\title{
Optical Waveguides and Resonant Cavities
}

\author{
Ana Vukovic, ${ }^{1}$ Martin Cryan, ${ }^{2}$ Snjezana Tomljenovic-Hanic, ${ }^{3}$ and Jun Shibayama \\ ${ }^{1}$ George Green Institute for Electromagnetic Research, The University of Nottingham, Nottingham NG7 2RD, UK \\ ${ }^{2}$ Department of Electrical and Electronic Engineering, University of Bristol, Bristol BS8 1UB, UK \\ ${ }^{3}$ School of Physics, The University of Melbourne, Parkville, VIC 3010, Australia \\ ${ }^{4}$ Faculty of Science and Engineering, Hosei University, 3-7-2 Kajino-cho, Koganei, Tokyo 184-8584, Japan
}

Correspondence should be addressed to Ana Vukovic, ana.vukovic@nottingham.ac.uk

Received 30 November 2010; Accepted 30 November 2010

Copyright (C) 2011 Ana Vukovic et al. This is an open access article distributed under the Creative Commons Attribution License, which permits unrestricted use, distribution, and reproduction in any medium, provided the original work is properly cited.

Optical waveguides and resonant cavities offer highly valuable ways of manipulating and processing light over short distances. Low loss propagation, efficient coupling to fibres, and ultracompact bends are paramount for creating lowcost photonics suitable for mass markets. Novel forms of waveguiding are being further exploited: photonic crystal waveguides have the potential for low loss guiding and exhibit slow light phenomena, and surface plasmon waveguides are increasingly used for sensing and imaging applications. Development of new devices is accelerated when strong links between research, development, and production are established, and in that process accurate numerical modelling is needed to reliably predict the performance and scope of a new device. This special issue reports on recent developments in design of photonic devices and addresses issues that need to be considered and approaches that need to be used for modelling purposes. In this special issue, we have invited a few papers that address these issues.

The first and second papers report on modelling and design of plasmonic-based devices. Plasmonic structures are very sensitive to design parameters and immediate environment and are ideal candidates for sensor devices. Furthermore, as plasmonics offers to integrate light with electronics on a single platform, large research is being conducted in the area of design and optimisation of a variety of optoelectronic devices. The first paper investigates two structures for the TM-pass/TE-stop surface plasmon polarizer. The first structure has a thin film sandwiched between periodic dielectric gratings. Numerical Finite Difference Time Domain (FDTD) method is used for analysis and optimisation and reports $94 \%$ transmission and an extinction ratio better than $17 \mathrm{~dB}$. The second structure addresses a more practical device in terms of fabrication in which a dielectric grating is sandwiched between two thin metal layers but reports reduced TM transmission and extinction ratio. The second paper compares the performance of various plasmonic waveguides and gratings using the Yee-mesh-based Beam-Propagation Method (YMBPM) and frequency-dependent FDTD method. Plasmonic waveguides are compared in terms of their effective index and propagation loss, whilst plasmonic gratings are compared in terms of their transmission spectrum. It is shown that the shape of the grating can greatly affect the transmission spectrum of a device.

The third paper considers modelling of photonic waveguides with oblique and tilted incidence in order to eliminate nonphysical staircasing noise that appears when Cartesian mesh is used. For this purpose, the oblique BPM method is implemented with Du-Fort Frankel scheme for fast computation. The implementation of the method is presented, and its accuracy and stability is compared with oblique CrankNicholson BPM method.

The fourth paper presents theoretical and numerical analysis of tunable photonic crystal coupled cavity waveguides for use in single photon transfer. The current challenge is to reduce the size of components for single photon manipulation by using either photonic crystals or plasmonics. The paper shows that useful performance could be achieved with coupled cavity waveguides and will act as an impetus to experimentalists in the field.

The fifth paper presents an improved design for CMOS compatible Electro-Optic (EO) modulator that combines lithium niobate and organic polymers on a silicon substrate. Combining these materials in the slot waveguide design 
enables tight concentration of both RF and optical fields for maximum interaction and reports on reduced RF losses making the modulator applicable for RF frequencies of up to $250 \mathrm{GHz}$. The hybrid modelling method is used whereby the modelling of the optical signal is done using the frequency domain FDTD method, whilst for modelling of the RF signal Finite Element Method with adaptive meshing is used in order to accurately describe both the subwavelength and larger features of the modulator.

The final paper investigates design, characterization, and realization of silicon microcavity photodetectors operating at $1.55 \mu \mathrm{m}$. The authors compare top- and back-illuminated configurations with a view to enhance the responsivity of the Schottky diode-based photodetectors.

\author{
Ana Vukovic \\ Martin Cryan \\ Snjezana Tomljenovic-Hanic \\ Jun Shibayama
}



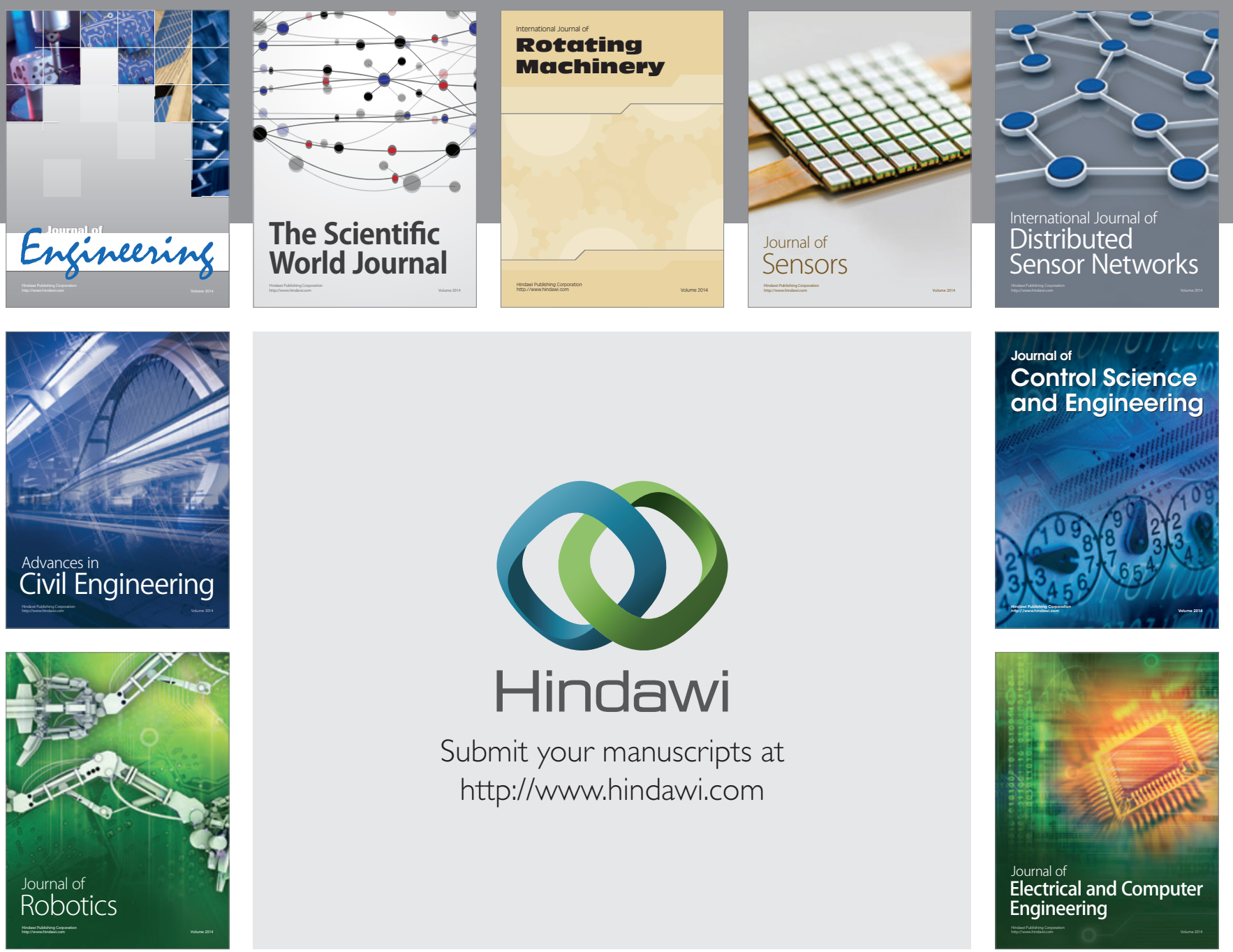

Submit your manuscripts at

http://www.hindawi.com
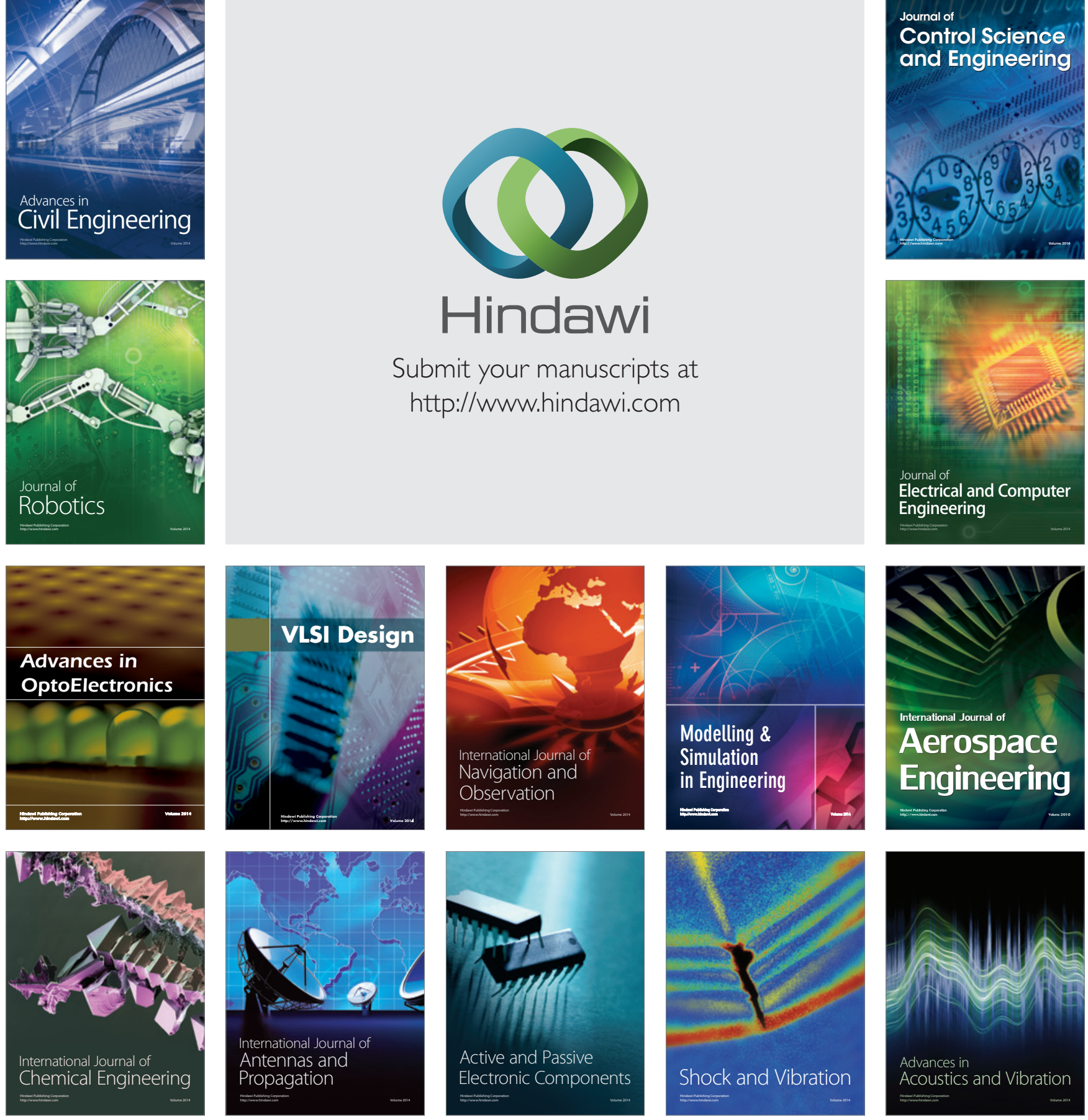